\title{
COMMUNICATION
}

\section{Non-invasive detection and chemical mapping of trace metal residues on the skin}

Cite this: DOI: $10.1039 / \times 0 \times x 00000 x$

\author{
S. M. Bleay, ${ }^{a}$ L.E. Grove, ${ }^{b}$ P.F. Kelly ${ }^{*^{c}}$, R.S.P. King $^{c}$, K. Mayse ${ }^{c}$, B.C. Shah $^{c}$ \\ and R. Wilson ${ }^{c}$
}

Received 00th January 2012,

Accepted 00th January 2012

DOI: $10.1039 / \times 0 \times x 00000 x$

www.rsc.org/

\begin{abstract}
Residual traces of the metals copper and lead on an individual's skin may be elucidated by reaction of rubeanic acid with a gelatine lift taken from the target area in question, yielding intensely coloured reaction products. This non-invasive technique has been shown to offer promise in chemically mapping the distribution of the minute metal residues across an individual's hand deposited through touching metal items, a technique with potential forensic significance.
\end{abstract}

Over the past few years, against a backdrop of general reductions in acquisitive crime, the frequency of metal theft offences has slowly risen. A major driving force of this type of crime may be attributed to increases in raw metal prices in response to global demand far exceeding supply. ${ }^{1}$ Against a backdrop of a more general downward trend in crime, Bennett ${ }^{2}$ and Haynes ${ }^{3}$ reported that metal theft has emerged as one of the fastest growing genres of crime within the United Kingdom, whilst Kooi ${ }^{4}$ provided evidence to show that steep increases in this crime type are also being observed in the Unites States of America. The UK ecclesiastical insurance office, for example, received almost 12,000 claims, at an estimated cost of more than $£ 30$ million, in 2007..$^{5}$ According to Bennett, the cost to the UK alone in 2008 was approximately $£ 360$ million; more recent figures from the Association of British Insurers suggest a scale in excess of twice this amount, despite some reductions attributed to the recent Scrap Metal Dealers Act. The economic and social impact of metal theft crime is extensive; it affects both national infrastructure (such as theft from telecommunication and railway companies) as well as national heritage. On the latter point, theft and damage to historic monuments and buildings can often result in collateral damage far in excess of the monetary worth of the material stolen (e.g. as a result of ensuing water damage etc.).

Despite efforts to implement various schemes to help reduce the occurrence of metal theft crime, such as property marking and Scrap Metal Dealers Act 2013, this area of illegality is still very much rife and of direct importance to both the police forces and the forensic practitioner. Bond et al. ${ }^{6}$ have recently acknowledged that advances in forensic analysis could perhaps pave the way for how metal theft crime scene exhibits are handled and processed. Their work focussed on the uniqueness of lead metal samples from church roofs, in a manner that would ideally provide intelligence as to the origin of any seized materials during investigation.

An important concept that has been little investigated within the discipline, however, is the notion of directly analysing the skin (hands) of a detained individual (suspect) for evidence pertaining to their recent activities. Field test kits exist that go some way into the detection of gunshot residue (GSR) $)^{7,8}$ and some forms of narcotic drugs $^{9}$ from an individual's hands or clothing; however in such cases evidence is gathered via 'swab' collection, limiting the accuracy of attempts to accurately map out the distribution of the material in question. Such mapping has the potential to add important strands to the forensic evidence associated with a case. Thus, in the example of metal theft, confirming the presence of metal traces on a suspect's hands would be only one desirable feature of the analysis; mapping its presence and distribution would prove an important adjunct, capable - in some scenarios at least - of linking the suspect to individual items.

The concept of recovering forensic evidence from the skin, or clothing, following handling of metal objects (such as weapons in the form of guns, wrenches, etc.) has, in part, been explored previously. The commercially available product, Ferrotrace, ${ }^{10,11}$ is a non-toxic iron detection spray that may be applied to the suspect's skin as a presumptive test for the localisation of iron residues resulting from dermal contact with a particular ferrous-based item. Building upon this, Lukaszewicz et al have developed various 1,2,4triazine chromogenic reagents for the detection of both ferrous and nonferrous metal traces left on contact with fabric and human skin. ${ }^{12}$ One distinct disadvantage of such methodologies, however, is the notion that the process is invasive to the individual concerned and photographic evidence must be acquired at the time of testing (as any developed evidential marks remain on the suspects skin and). Lukaszewicz went some way in trying to alleviate such an invasive process through the application of triazine-soaked cotton swatches to the target area in question, thereby transferring an appreciable amount of iron residues to the swab and only minimally staining the 
skin. ${ }^{13}$ The development on the transfer medium in these instances showed substantial 'bleeding' as a result of the swatch material type, thereby prohibiting intricate detail, such as that required for fingerprint identification, to be obtained. Moreover, such a process still stained the individuals skin to some degree and best results occurred only when metal-to-skin or metal-to-fabric contact exceeded several tens of minutes in duration. A non-invasive variation of the iron reagent testing protocol has also been outlined by Lee through the transfer of residues on skin to wet filter paper. ${ }^{14}$

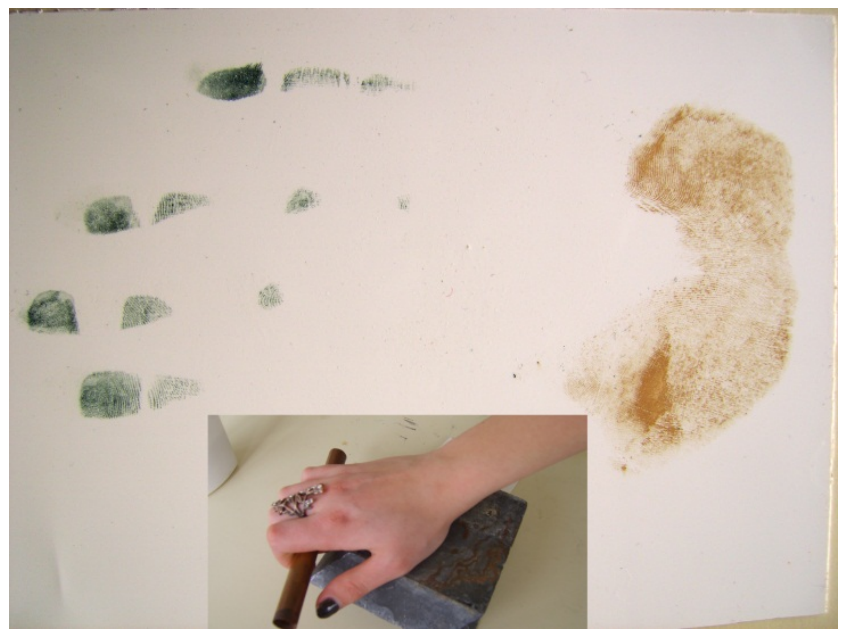

Fig.1 The effect of rubeanic acid treatment of a gel-lift from a hand that has briefly held a copper pipe while touching a lead brick (inset).

In the work reported here, the metals examined were copper and lead, chosen both for their relevance to metal theft scenarios (i.e. as the bulk metal) and to heritage crime (as components of artefacts made from the metals themselves of from appropriate alloys such as bronze). Adhesive gelatine sheets were employed in order to access the traces of metal deposits from skin, while at the same time negating any need for direct chemical contact. Though such sheets are routinely used to "lift" and store developed fingerprints from scenes and to retrieve evidence such as footmarks, they appear to have found much less use in terms of direct application to skin. Indeed, in a more general sense the notion of sequential treatment of these 'lifting' materials with complimentary chemical processes, that may then be able to provide additional evidence relating to the crime in question, appears surprisingly neglected.

The final component of the technique is the developing agent, in this case rubeanic acid (dithiooxamide, $\mathrm{H}_{2} \mathrm{NC}(\mathrm{S}) \mathrm{C}(\mathrm{S}) \mathrm{NH}_{2}$ ). This is a polydentate ligand that can chelate to metal ions via both its nitrogen and sulfur atoms. Interaction of copper ions with this ligand has been the subject of structural studies and has found forensic utilisation. Thus, for example, rubeanic acid is employed in techniques that determine the distribution of copper remnants from bullet firings in order to ascertain firing distances. ${ }^{15}$ Very recently, an intriguing case report details the use of rubeanic acid in linking a cutting tool (a sickle) with stolen telephone cable enclosing numerous pairs of copper wires. ${ }^{16}$ The copper/rubeanic acid interaction has been studied away from the forensic arena and has been shown to result in the formation of a polymeric complex of the ligand (as per Scheme 1) which is the imaging agent in the forensic techniques. ${ }^{17}$

Interaction of rubeanic acid with lead has been less studied, with reports that while a yellow complex initially forms, it decomposes to lead sulfide. ${ }^{18}$ Likewise, reaction of an alkaline solution of rubeanic acid with lead (II) hexacyanoferrate in a gelatine matrix was shown to yield a black precipitate of lead sulfide. ${ }^{19}$ In the case of the gels treated in this current study, the darkening upon treatment with UV is consistent with formation of a lead complex which in turn is sensitive to UV light. The final colour, however, is not consistent with complete degradation to lead sulfide. In fact, in our hands exposure of lead acetate/rubeanic acid mixtures (stirred in ethanol) to UV resulted in the formation of a brown precipitate similar in colour to the "developed" trace marks on the gels. The infra-red spectrum of this precipitate clearly exhibits key bands associated with rubeanic acid, while microanalysis results show appreciable carbon and nitrogen levels that are, nevertheless, slightly low for a lead analogue of the copper product shown in Scheme 1 (C 6.4, $\mathrm{H}$ 0.5, N 6.9\%; calc for $\mathrm{PbC}_{2} \mathrm{~N}_{2} \mathrm{H}_{2} \mathrm{~S}_{2}$ C 7.4, H 0.6, $\mathrm{N}$ 8.6\%). The most likely explanation is that the previously reported lead complex is indeed forming, with its pale yellow colour making immediate reaction on such a small scale invisible on the gel until UV-induced degradation begins.

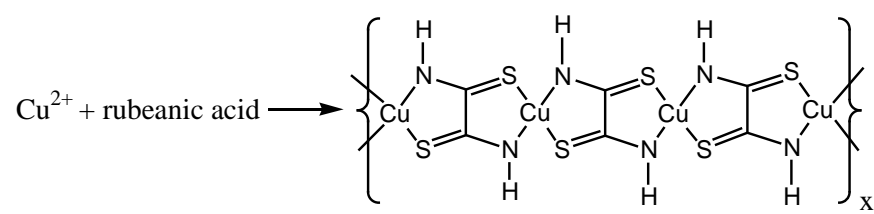

Scheme 1 The formation of the polymeric complex of $\mathrm{Cu}^{2+}$ with rubeanic acid. ${ }^{17}$

The overall process in question is well illustrated by Fig. 1. In this case a subject's hand is in contact with two metal samples - a copper pipe and a lead brick. After a few seconds of contact time and then removal of the hand from the metal, a gelatine "lift" from the palm was taken and the sheet treated with a $0.1 \% \mathrm{w} / \mathrm{v}$ solution of rubeanic acid in absolute alcohol within a standard dipping tray. Evidence for the presence of copper at the end of the fingers was manifest immediately by the dark blue/black colour apparent in Fig.1. $\dagger$ Subsequent exposure of the sheet to UV light brought about the development of the brown colouration at the base of the palm area, indicative of the presence of lead. The key point about the image in Fig. 1 is the distinct coloration associated with the two metals and clear delineation between areas of the hand which had touched either metal or indeed those areas which touched neither and thus appear blank on the developed sheet.

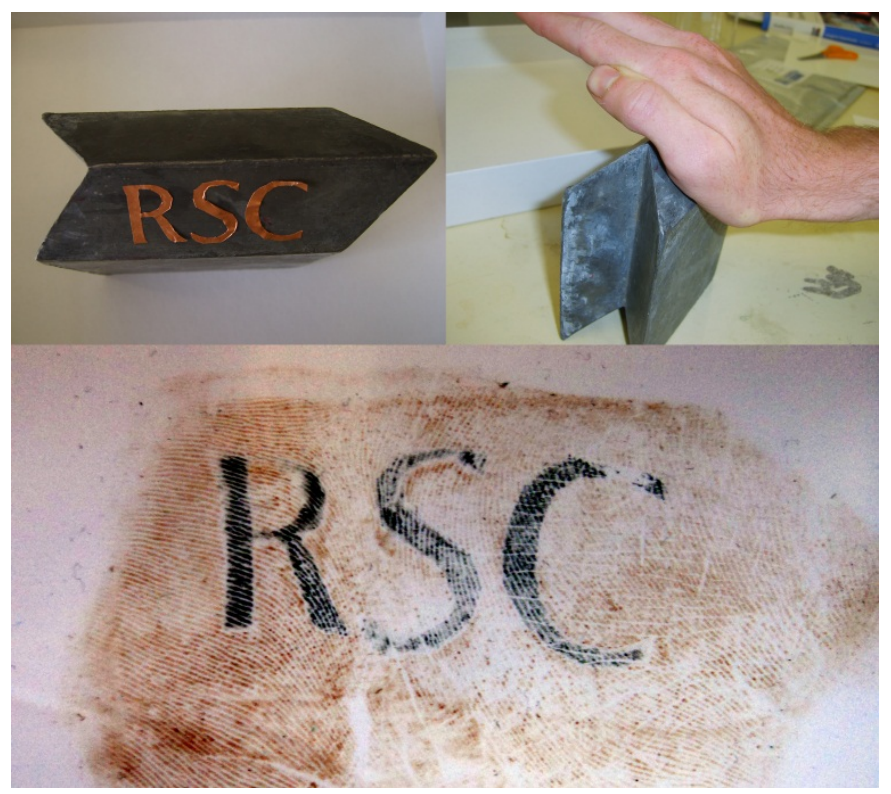

Fig.2 The effect of rubeanic acid treatment of a gel-lift from a hand that has briefly touched (top right) the copper logo on a lead brick (top left). 
Importantly, this delineation can map the metal distribution to a high level of accuracy. This is illustrated in Fig.2; here the logo of the RSC is picked out in copper metal on the surface of a lead brick. Now, after a brief contact time, and subsequent sampling via gelatine sheet, development as before clearly leads to the appearance of the copper logo distinctly differentiated from the lead background and the untouched areas.

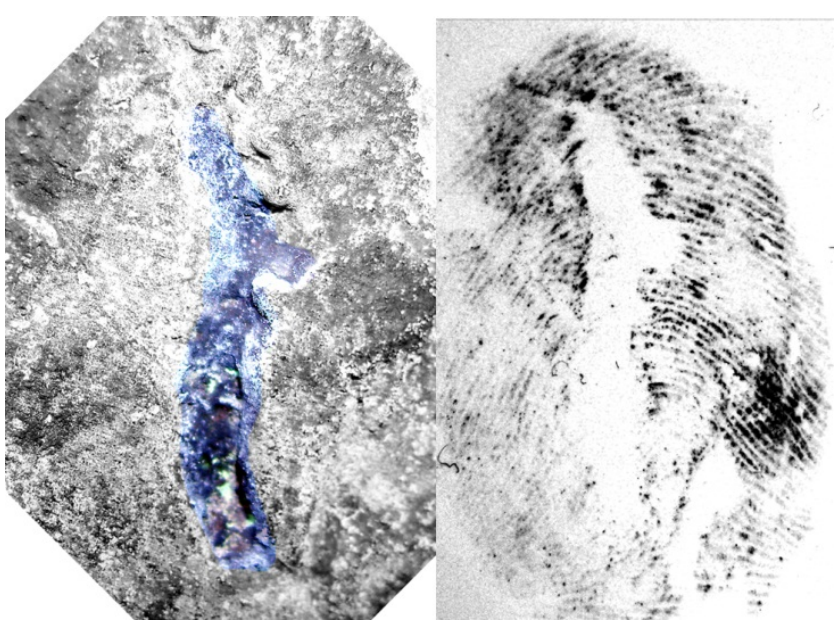

Fig.3 (left) a deformation (c.a. $2 \mathrm{~cm}$ in length) in the surface of a weathered bronze statue, highlighted in blue; (right) corresponding image developed from a gel-lift off a thumb briefly placed over the area in question.

The ability to link precise distribution to potential artefacts has obvious potential with regard to handling heritage items. Thus in Fig.3 we see that slight surface irregularities in a weathered bronze sculpture are mirrored in the distribution of copper on the thumb that touched it, thereby linking that copper presence to contact with the item in question. This has particular potential with regard to items for which attempts to obtain fingerprints might be undesirable from aesthetic or conservation perspectives.

As a potentially viable forensic technique this process benefits from a number of features. Firstly, the application of the rubeanic acid solution can be performed via a hand-held spray; thus results could be easily obtained in situ at areas of investigation (though in the case of the lead development, a UV source would also be required to expedite the process - without UV treatment the lead images take a number of hours to appear). Solutions appear active for many weeks when kept in a light proof spray bottle, obviating the need to make up the active rubeanic acid reagent "freshly" for each application. Another practical advantage of the technique comes from the fact that once the gel "lift" has been taken from a hand, the results can be stored and then developed with the rubeanic acid many days later. In addition, an analogous effect is not apparent having touched precious metals, nickel, aluminium or steel surfaces. Another pertinent feature of this technique is the fact that results can be obtained many hours after contact with the metal. This extends much longer in the absence of washing the hands, while even after light washing useable results are still apparent. Indeed, the latter observation provides some hint as to the chemical nature of the metal species transferring to the hand during such brief contact. In the copper case at least, this would appear most likely a combination of both oxidised and elemental metal. The fact that gel lifting from a hand that has been immersed in water for a minute after touching a copper surface generates similar - though less intense - results after development, indicates that even though soluble $\mathrm{Cu}(\mathrm{II})$ will have been removed, the effect persists. Thus it would seem most likely that both $\mathrm{Cu}(0)$ and $\mathrm{Cu}(\mathrm{II})$ (either present on the metal surface already of formed via oxidation through the action of sweat) are the "active" species transferred during contact. It is also noteworthy that in addition to the visualisation of the metal associated with an individual's palm print, the "developed" gelatine lifts are also amenable to further analysis. Preliminary work has shown that Laser Ablation Inductively Coupled Plasma Mass Spectrometry (LAICPMS) can be effectively employed on the resulting samples, which gives rise to the possibility of precise isotope analysis or identification of species present at too low a level for chemical imaging. $\dagger$

\section{Conclusions}

In conclusion, this technique has obvious potential in terms of assisting in the examination of metal theft suspects, given that the process can link an individual to a particular metal source based on the colour of the developed handprint and the pattern of metal distribution across the individual's hand. The process is highly flexible as it can be used in the forensic laboratory or in situ as spray solutions, is non-invasive, can still generate results a significant time after contact with metal and after light washing, needs only a few seconds of contact with the metal and has given meaningful results with all donors thus far tested (albeit with the absolute intensity of the developed colouration sometimes varying from donor to donor). The level of detail that can be mapped out indicates that the technique has particular potential within heritage crime scenarios e.g. the opportunistic theft of smaller, high value antique items.

\section{Notes and references}

${ }^{a}$ Home Office Centre for Applied Science and Technology, Sandridge,

St. Albans, United Kingdom.

E-mail: Stephen.Bleay@homeoffice.gsi.gov.uk; Tel: +44 (0) 1727816252

${ }^{b}$ Department of Social Sciences, Loughborough University,

Loughborough, LE11 3TU, United Kingdom.

E-mail: l.e.grove@lboro.ac.uk; Tel: +44 (0) 1509564017.

c Department of Chemistry, Loughborough University, Loughborough, LE11 3TU, United Kingdom.

E-mail: P.F.Kelly@lboro.ac.uk, R.King@lboro.ac.uk;

Tel: +44 (0) 1509222578.

$\dagger$ Original digital images (Samsung Digimax V800 camera) from the developed gel-lifts for Figs. 1 and 2 were processed using the "Autolevels" feature of Photoshop Elements 2.0. An example of the original image is shown in the ESI.

†† The authors acknowledge B.L.Sharp, G.Craig and A.J.Managh, Department of Chemistry, Loughborough University, for help in running preliminary LA-ICPMS studies.

Electronic Supplementary Information (ESI) available: Experimental information. See DOI: 10.1039/c000000x/

1 A. Sidebottom, J. Belur, K. Bowers, L. Tompson and S.D. Johnson, J. Res. Crime Delinq., 2011, 48, 396-418.

2 L. Bennett, Environ. Law Manage., 2008, 20, 176-83.

3 C. Haynes, Jane's Police Review, 2008, 18-21.

4 B. Kooi, U.S. Department of Justice, Office of Community Oriented Policing Services, 2010, Guide No. 58.

5 Ecclesiastical Insurance Office. Guidance notes theft of metal, http://www.ecclesiastical.com/Images/PDF\%20-

\%20Theft\%20of\%20metal\%20guidance\%20notes.pdf (accessed October 31, 2013).

6 J.W. Bond, S.V. Hainsworth and T.L. Lau, J. Forensic Sci., 2013, 58, 1003-1007.

7 P. Weklinski, Evidence Technology Magazine, 2004, 2, 28-31.

8 F.S. Romolo and P. Margot, Forensic Sci. Int., 2001, 119, 195-211. 
9 Field test kits for narcotics may be purchased from numerous suppliers, see for example Westminster International Ltd.

10 Ferrotrace is supplied by Arrowhead Forensics

11 J. Almog, J. Forensic. Sci., 2006, 51, 1228 - 1234.

12 A. Olejniczak, A.W. Cyganiuk and J.P. Lukaszewicz, J. Forensic Sci., 2010, 55, 747-752.

13 J. Szumera, M. Welniak, A. Olejniczak and J.P. Lukaszewicz, J. Forensic Sci., 2010, 55, 944-952.

14 C.-w. Lee, J. Forensic Sci., 1986, 31, 920-930.

15 B. Glattstein, A. Vinokurov, N. Levin and A. Zeichner, J. Forensic Sci., 2000, 45, 801-806.

16 R. Kumar, N. Patial and S. Singh, J. Forensic Sci., 2013, 58, 217219.

17 M. Abboudi, Inorg. Chem., 1985, 24, 2091-2094

18 P. Ray and J. Xavier, J.Indian Chem.Soc., 1961, 38, 535-555.

19 O. Mikhailov, Russ. J. Coord. Chem., 2003, 29, 276-280. 